\title{
New Approaches to Design Practice
}

\author{
Douglas MacLeod \\ Canadian Design Research Network \\ Simon Fraser University \\ Central City \\ 250 - 13450 102nd Avenue \\ Surrey, BC V3T 0A3 \\ dmacleod@cdrn.ca
}

\begin{abstract}
Contemporary design practice is both borrowing from and contributing to other disciplines. This paper will examine two significant trends - design-based research and evidence-based design-that will have an impact on current and future design practices. Design-based research is currently popular in the field of education and borrows from the way architects and engineers work to develop their ideas in real-world contexts and as such it provides a new perspective into design engineering itself. Evidence-based design borrows from work done in evidence-based medicine to carefully observe and analyze the way people use the products of design. Taken together these two trends provide a new approach to design and a better means of connecting with collaborators, consumers and users.
\end{abstract}

\section{Introduction: "Culture is Everything”}

The world of design is changing. New technologies and new realities are forcing design professionals to re-examine their traditional approaches to practice. Broadband networks and interactive multimedia provide new channels for enhanced communications, the testing of multiple alternatives, and the collection and analysis of data. In turn these developments suggest that our traditional ideas about design and research must evolve as boundaries and responsibilities blur. Even the concept of design engineering suggests that the distinctions between designers and engineers are breaking down.

Simultaneously other disciplines are taking an active interest in design and design thinking. According to advocates such as Roger Martin, Dean of the Rotman School of Management at the University of Toronto, design thinking promotes the idea of abductive reasoning which combines inductive and deductive thinking to identify promising (but not confirmed) theories and explore them rigorously as a means of refinement [1]. In effect abductive reasoning investigates, then modifies, a hypothesis by applying it to a real world situation. Abduction is "good fit" with how humans actually approach problem solving.

Within this context two new approaches will be examined - one from within and one from outside design. Evidence-based design is developing within the design professions as a means of bringing greater rigor to design decisions. Design-based research, on the other hand, has borrowed from the world of design and is being applied to areas such as education and business. The intent is to show both how design practice is evolving from within (through evidencebased design) and how design is perceived from outside the design professions (through design-based research). This analysis will then be used to synthesize some generalized trends about design and design research.

It is important to note that these new ideas bring with them a distinct set of values and attitudes that must be made explicit. IDEO, one of the most successful contemporary design firms, recognizes this when they proclaim, "Culture is everything [2]." Participatory, interdisciplinary, iterative and collaborative are some of the characteristics that have been attributed to these new methodologies and to this evolving design culture, but each of these qualities can have both a positive and negative effect on designparticularly when they come in conflict with traditional means of practice. While this new found appreciation of design is on the whole a positive development, an uncritical embrace of design thinking can lead to difficulties and new techniques should be 
carefully evaluated before they are implemented on a large scale.

\section{Background}

The first generation of Computer-Aided Design (CAD) packages were little more than electric pencils that imitated manual drafting. As these applications became more powerful they began to suggest other approaches to design. In particular, they began to employ increasingly sophisticated data structures that would allow the designer to develop and analyse multiple alternatives in much the same manner that a spreadsheet allows its user to map out a variety of "what if?" scenarios. Current interest in Building Information Modeling or BIM is the latest manifestation of this trend in which building data can

\section{Design-Based Research}

Design-based research is an example of this trend which is gaining prominence in fields such as education. According to its proponents,

Design-based research is a relatively new methodology developed by and for educational researchers. It is interventionist - in that a purposeful change is made in a functioning educational context. It is participatory in that researchers work with practitioners. It is theoretical in that theory is used to design the intervention. Further, it generates theory through studied application in the original and subsequent educational contexts in which the innovation is used. [4]

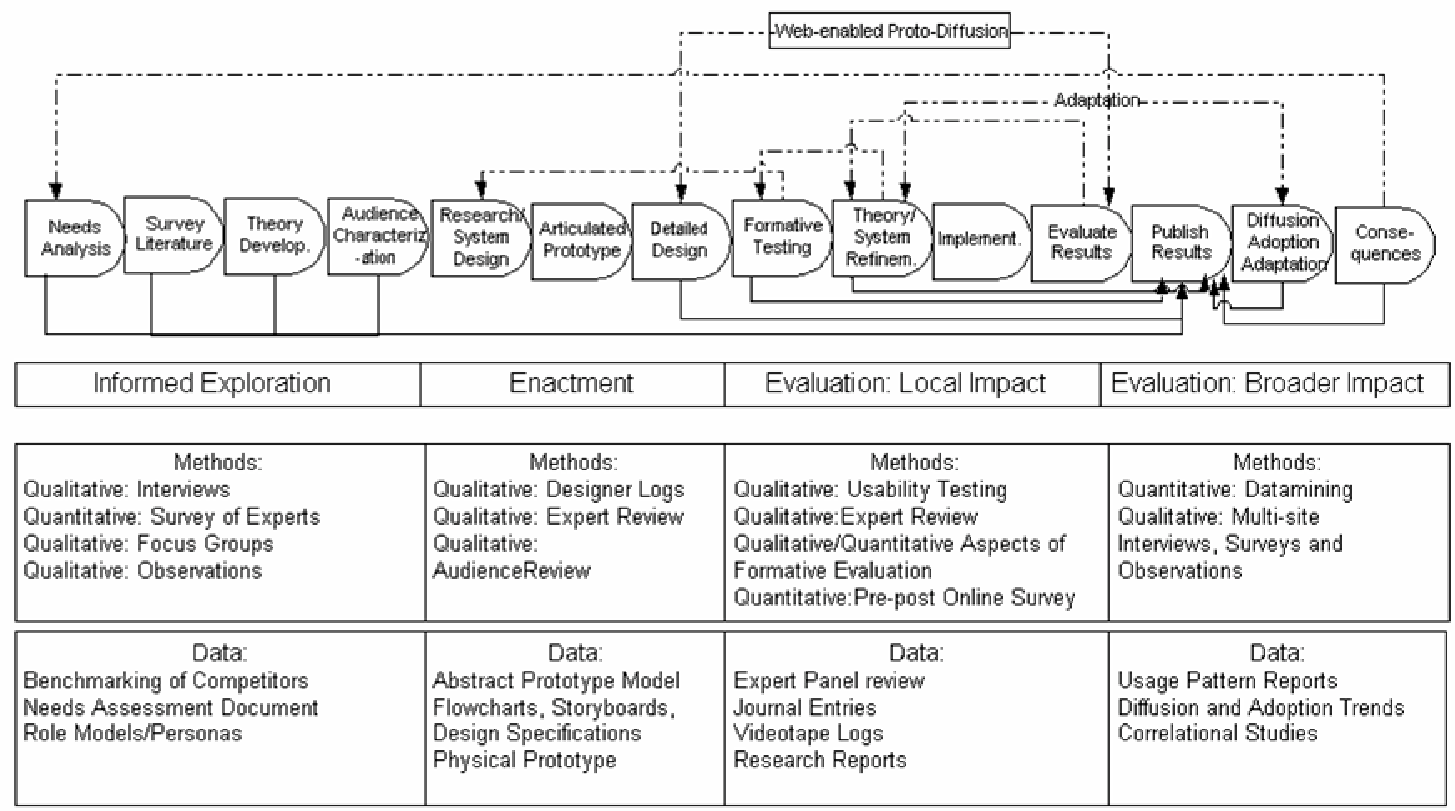

be used, reused and exchanged quickly and easily among all members of the design team.

This use of structured information coupled with the increasing speed with which all forms of information can be shared across vast distances through the Internet may partially account for the new interest in design. According to Martin, "If indeed the design economy is emerging as the successor to the information economy, it is in part because the information economy has created efficiencies and productivity that have provided an opportunity for a next wave of value creation [3]."
Figure 1. Merging of design and research processes into the integrative learning design framework (Bannan-Ritland)

While design-based researchers acknowledge that this idea is borrowed from the disciplines of architecture and engineering [5] they take it far beyond these two disciplines. The Design-Based Research Collective, for example, suggests that, “ ... in design-based research, practitioners and researchers work together to produce meaningful change in contexts of practice. [6]" Barab and Squire add that " ... design-based research involves flexible design revision, multiple dependent variables, and capturing social interaction. [7]" Bannan-Ritland has elaborated on these ideas to propose a full-fledged, step-by-step methodology which is reproduced in Figure 1 above 
[8]. Significantly, her schematic includes multiple feedback loops where rigorously gathered evidence is used to improve the design at a number of steps during the process.

Many of these attributes - such as researchers and practitioners working together, capturing social interactions and feedback loops - should also inform and be incorporated into our own models of research and practice. What is most revealing, however, is the way these researchers perceive design. As Barab and Squire note, "One challenging component of doing educational research on design-based interventions is to characterize the complexity, fragility, messiness, and eventual solidity of the design and doing so in a way that will be valuable to others. [9]" [Emphasis mine].

To outsiders design does appear to be messy, complex and fragile but this confusion also suggests a lack of understanding of the design process that is also reflected in the Bannan-Ritland schematic. In particular two aspects of the design process are missing in design-based research - precedents and brainstorming.

Precedents are particularly important. Over the course of some 6000 years of building, architects and engineers have built up a repertoire of successful designs from which they draw - often without even realizing it. The books, journals, catalogues, slide collections and libraries of details that are part of any professional design practice all provide evidence of this approach. Those who wish to emulate design need to establish similar collections of precedents.

These libraries, however, must be built up and used with care. In design, precedents act more as means of inspiration rather than a recipe or a kit of parts to be religiously followed. In fact, and this is a crucial aspect of design culture, precedents when re-worked and re-packaged are most often used as a vehicle for doing things in a different way.

Brainstorming is another tool for creative innovation that is another essential part of design culture and it too is missing from the Bannan-Ritland model. Brainstorming has also been termed "Serious Play" by Michael Schrage in his book of the same name and he provides the following definition:

It means innovation requires improvisation. It means innovation isn't about rigorously following "the rules of the game" but about rigorously challenging and revising them. It means innovation is less the product of how innovators think than a byproduct of how they behave. Serious play is about innovative behavior [10].

IDEO, the industrial design firm mentioned earlier, has institutionalized brainstorming as part of their methodology (which is reproduced $n$ Figure 2 below).
They have even developed seven rules for brainstorming which are stenciled on the walls of their studio: "Be Visual. Defer judgment. Encourage Wild Ideas. Build on the Ideas of Others. Go for Quantity. One Conversation at a Time. Stay Focused on the Topic. [11]"

Brainstorming is also implicit in the schematic design phase of an architectural project. Since for designers these are often the most enjoyable stages of the work, their omission from the design-based research model is a significant one. It is, however, a flaw which could be easily addressed by simply adding it to the Informed Exploration phase where it would serve as a complement to the proposed needs analysis and benchmarks.

It should also be noted that the Bannan-Ritland model does bring a degree of rigor to design-based research the lack of which has been noted by other critics. Shavelson et al, referring to what they call "design studies," note that " ... many of these studies rely on narrative accounts to communicate and justify their findings. Although narratives often purport to be true, there is nothing in narrative form that guarantees veracity. [12]" A similar criticism, of course, could be applied to design in general where decision are often made on the basis of anecdotal evidence.

The same article provides a prescription for addressing this concern which again has relevance for all forms of design:

"To ensure that they are scientifically rigorous, it is incumbent on those carrying out design studies to create a culture of science that includes ruling out competing hypotheses, that foster skepticism about knowledge claims (including their own), and encourages powerful tests of rival conjectures. [13]"

It is precisely this type of rigor that is embodied (or should be embodied) by evidence-based design.

\section{Evidence Based Design}

A number of design professionals are investigating the idea of evidence-based design as a means of meticulously examining past buildings in order to build better new ones. Evidence-based design borrows from work done in evidence-based medicine to carefully observe, quantify and analyze the way people use buildings.

The Farrow Partnership Architects Inc. of Toronto is currently using evidence-based design techniques on a number of health care facilities - most notably the Carlo Fidani Peel Regional Cancer Centre in the Credit Valley Hospital.

With a $\$ 100,000$ research grant from the Ontario Health Association Change Foundation and another $\$ 100,000$ in an in-kind contribution of labour, the 
design team was able to measure, evaluate and learn from the performance of a similar facility at the Grand River Hospital.

A rigorous methodology of the kind suggested by Shavelson is critical to evidence-based design. In the multivariable context of any environment the careful collection of data is essential in determining if the architecture really is affecting the health of the patient - rather than some related but less obvious factor. To this end, Karen Parent, an Assistant Professor in the Faculty of Physical Medicine and Rehabilitation at Queen's University and the President and CEO of Workflow Integrity Network, established the framework in which the research could occur and in which valid data could be collected.

The team was able to observe 10 nurses and approximately 60 patients in the Grand River facility and is now observing the same patients and nurses in the new facility. This is a very unique (if rare) situation that helps isolate the design from other variables.

Parent's team observes the patients and staff from a distance and (as unobtrusively as possible) enters their observations into their hand-held palm pilots. These palm pilots were pre-programmed with a variety of daily activities that would occur in the clinic and the observers would select the activities as they occurred and enter pertinent data such as travel time and contacts. In effect the design team built a database of inputs and outputs and most recently they have collected (but not yet analysed) six months worth of data from the new facility at the Credit Valley Hospital. This case study emphasizes the fact that when evidence-based design studies are conducted properly they are both time-consuming and expensive. While undoubtedly valuable, evidence-based design cannot be divorced from the economic realities of a design practice. Most clients would be reluctant to incur additional costs for such studies (no matter how valuable) and $\$ 100,000$ of in-kind labour represents a significant contribution from a small or medium-sized design firm.

Creating the warrants of knowledge through the careful analysis of the collected data is also a daunting task. Some of the studies prepared by the Heschong Mahone Group give ample evidence of this [14].

For example, a study on "Daylighting in Schools" conducted in 1999 by George Loisis in California found that in San Juan Capistrano students with the greatest amount of daylighting in their classrooms improved $20 \%$ faster on math tests and $26 \%$ faster on reading tests (between fall and spring testing) than those students in classrooms with the least amount of daylighting. [15]
The Heschong Mahone Group, however, could not duplicate the San Juan Capistrano results in Fresno, California and found that the Daylight Code, a holistic variable used to rate classrooms by the amount of light they receive, was not significant in Fresno schools, In fact, in a number of cases a high Daylight Code was found to have a negative impact on student performance.

Investigating further the team “ ... found the classrooms with high daylight codes to have reverberation levels above current national recommendations [16]." In other words acoustics and daylighting (not daylighting alone) combined to affect learning performance.

Moreover when all other factors were taken into account the group was forced to conclude that "the physical characteristics of classroom [sic] have a very minor potential influence on the performance of a given individual ... [17]" - although they did feel that even this minor benefit extended across the life of a school and affecting hundreds of students was well worth pursuing.

Like design-based research, evidence-based design requires a rigor and an analytical sophistication that has not previously been part of design practice. This raises a number of important questions about design itself.

\section{Re-Designing Design}

These new approaches to design are often predicated on the assumption that design studios are run on principles of interdisciplinary, egalitarian collaboration. This is simply not true. While a building project may be designed by multiple disciplines, there is a rigid hierarchy of responsibilities with the architect traditionally assuming a leadership role. Moreover, none of the proponents of design thinking seem to acknowledge the role of ego in design. While evidence-based design may modify this situation, design is still very subjective and individual designers often dominate the creative process to the exclusion of all others. It is difficult, for example, to imagine Frank Lloyd Wright engaging in participatory design and it is difficult to imagine the his greatest works could have emerged from such a process. Indeed some designers are fearful of techniques such as evidencebased design because they may constrain the creative process. For these new methods to realize their potential both design and designers must evolve.

In this respect IDEO has been cited throughout this paper as a design firm that does do things differently and there is no doubt that IDEO has created an intriguing perspective on design through a collaborative approach that relies on a unique process. 
This is what Mike Janes, Director of Operations in their San Francisco office refers to as a "blurring" of the disciplines [18]. He emphasizes their team approach which informs their hiring practices and generates respect for everyone involved. According to Janes, "People work so closely together on these teams that after six months it's hard to tell who's doing what [19]."

One reason that IDEO is successful is that they treat their clients with a similar respect. As shown in Figure 2 [20], each project begins with a research stage (which is essentially evidence-based design) that involves the first-hand observation of actual users. This phase is led but not dominated by human factors specialists. This is followed by a period of brainstorming which has been described earlier.

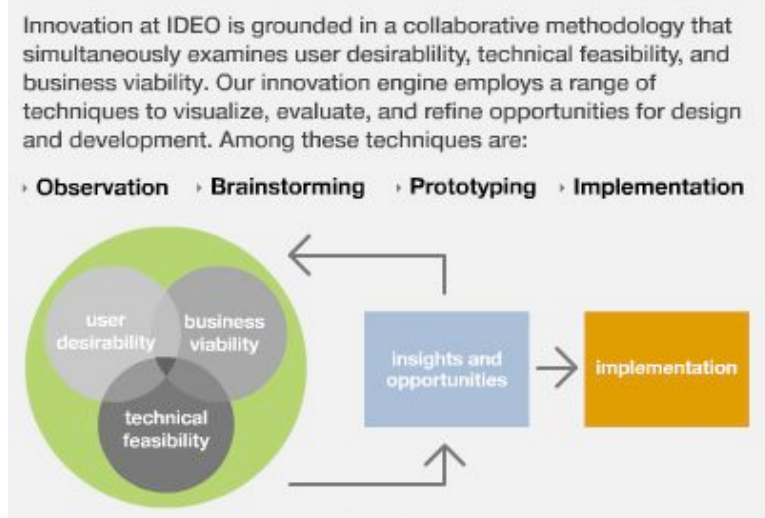

\section{Figure 2. IDEO Methodology}

Next comes prototyping which may range from conceptual models to functional mock-ups. It is informed by the desire to "fail early to succeed sooner [21]." And finally there is the Implementation phase where the detailed design and engineering is completed, manufacturers are brought on board (if necessary) and the product launch is planned.

There is no doubt that this user-centred approach is interdisciplinary and it does lead to innovative designs and an effective and respectful use of their human resources. Nonetheless, closer inspection shows that it improvises on top of a traditional system of design culture. Like an architecture firm, the studio and department heads meet once a week to organize production; quality levels are monitored and enforced if necessary; and designers are expected to work overtime without compensation. On the other hand, it has two important differences from an architecture firm. Most jobs take only ten to twenty weeks to complete which makes them easier to manage and finance and, perhaps most important of all, they handoff the intellectual property rights of their designs to the client. Because the client owns the intellectual property, they also own the liability. With the reduced cash flow of shorter projects and the reduced cost of carrying liability insurance, IDEO can effectively outbid and out-perform more traditional firms.

Again, liability is not addressed by those who promote design thinking. What if an evidence-based post-occupancy study of a building reveals serious flaws in its performance? Does an anthropologist on an interdisciplinary design team need to carry the same liability insurance as an engineer? Do communities that engage in participatory design exercises assume some of the risk? Serious study needs to be given to the mechanics of $21^{\text {st }}$ Century design.

In particular as design becomes more data driven, and more and more evidence is gathered, how will that data be archived and managed in a manner that is consistent, comprehensive, accessible and easy to use? Creating such an architecture of design information is a critical design challenge that involves practitioners, researchers, users and government agencies.

Moreover, how will such information find its way into practice? Organizations such as CDEN and the CDRN (Canadian Design Research Network) need to pool their resources to ensure that their work is shared with all relevant stakeholders.

\section{Summary: Culture Really is Everything}

The most disturbing aspect of the recent popularity of design, however, is that it may reduce design to just another business fad such as Total Quality Management at a time when design may prove essential to Canadian prosperity. Martin is right, design is a competitive advantage and, as noted in other papers in this collection, other nations are investing heavily in design and building national design strategies. Canada must do the same.

In order to facilitate that process, however, new approaches to design such as design-based research and evidence-based design must be backed by robust support structures, a commitment by all of the participants and the necessary mechanisms (whether they be technologies or processes) to help them succeed. Designers (and non-designers) must understand the cultures involved and improvise and innovate on top of these systems as IDEO does. The potential of such a inclusive and integrative approach could be significant. When, regarding design studies, Shavelson and his team comment, “... we believe that coupling scientifically warranted knowledge and rich contextual information in some narrative form might lead to increased understanding and use of scientific research in practice [22]," they might well be describing the future of design itself. 


\section{References}

[1] Breen, B., "The Business of Design," Fast Company, April 2005, p. 69

[2] Janes, M., Telephone interview, April 1, 2003.

[3] Young, P., "Respect" in Applied Arts Magazine, Volume 21, No. 3, p. 31.

[4]_Accessed at cider.athabascau.ca/CIDERSIGs/

DesignBasedSIG/ on March 1, 2006.

[5] Anderson, T. Design Based Research PowerPoint,

November 2004. Accessed at

cider.athabascau.ca/CIDERSIGs/DesignBasedSIG/ on June $13,2006$.

[6] The Design-Based Research Collective, "Design-Based Research: An Emerging Paradigm for Educational Inquiry," Educational Researcher, Volume 32, No. 1, p. 6.

[7] Barab, S., and K. Squire, "Design-Based Research:

Putting a Stake in the Ground," The Journal of the Learning Sciences, Volume 13, No. 1, p. 3.

[8] Bannan-Ritland, B., "The Role of Design in Research: the Integrative Learning Design Framework," Educational Researcher, Volume 32, No. 1, p. 22.

[9] Ibid, p. 4.

[10] Schrage, M. Serious Play, Harvard Business School Press, Boston, 2000, p.1.

[11] Accessed at

www.ideo.com/about/methods/info.asp? $\mathrm{x}=2$ on June 15 , 2006.

[12] Shavelson, R.J., D.C. Phillips, L. Towne, and M.J.

Feuer, "One the Science of Education Design Studies"

Educational Researcher, Volume 32, No. 1, p. 25.

[13] Ibid, pp. 27-28.

[14] Accessed at http://www.h-m-

g.com/projects/daylighting/projects-PIER.htm on June 16, 2006.

[15] Ibid.

[16] Ibid.

[17] Ibid.

[18] Janes, M., Telephone interview, April 1, $2003 .$.

[19]Ibid.

[20] www.ideo.com/about/methods/info.asp? $x=2$

[21] Ibid.

[22] Shavelson, R.J., D.C. Phillips, L. Towne, and M.J. Feuer, "One the Science of Education Design Studies"

Educational Researcher, Volume 32, No. 1, p. 28. 